Ann. Génét. Sél. anim., I980, 12 (4), 363-376.

\title{
Influence d'une modification de l'accroissement des dépôts adipeux chez la poule pondeuse : Résultats d'une sélection divergente portant sur trois générations
}

\author{
B. LECLERCQ, J. C. BLUM et J. P. BOYER \\ Avec 1a collaboration technique de Gérard GuY \\ Station de Recherches Avicoles, I.N.R.A., \\ Nouzilly, 3738o Monnaie
}

\begin{abstract}
Résumé
Pendant trois générations, deux lignées de poules pondeuses de type Rhode Island sont sélectionnées pour un gain de poids relatif pendant la ponte, élevé ou faible. Les deux lignées répondent bien à la sélection mais l'héritabilité de ce caractère diminue rapidement. Le poids vif adulte et les performances de ponte ne sont guère modifiées au cours de trois générations. Aucune corrélation significative ue peut être trouvée entre l'état d'engraissement et les performances de ponte. Chez les poules de la lignèe maigre, l'efficacité alimentaire tend à être meilleure que chez celles de la lignée grasse; les différences ne sont cepenđant pas très prononcées. L'Association d'une sélection sur le gain de poids et sur l'indice de consommation semble la voie la meilleure pour améliorer l'efficacité alimentaire de la pondeuse.
\end{abstract}

\section{Introduction}

La poule pondeuse tend à s'engraisser au cours de la période de ponte. Mais ce phénomène présente une grande variabilité; en fait, certains animaux utilisent en partie leurs réserves adipeuses tandis que d'autres les augmentent notablement, ce qui se traduit par un accroissement du poids vif initial qui peut atteindre 25 p. IOO.

Nous avons pu démontrer que la variation du poids vif de la poule en ponte est un excellent indice de 1'évolution de ses réserves grasses (LECLERCQ, I975; LEOLERCQ et al., I977). Par ailleurs, on sait que cette variation du poids vif, si elle est mesurée dans des conditions précises, présente une héritabilité significative (BORDAS et MERAT, I976; LECLERCQ et al., I977). Nous avons entrepris 
la sélection divergente de deux lignées, l'une grasse, l'autre maigre en se fondant sur les variations de poids vif de la population sur laquelle portait notre étude initiale (LECLERCQ et al., 1977). L'objectif était d'étudier les répercussions d'une telle sélection sur les autres paramètres de la période de ponte (performances de reproduction, consommation d'aliment...).

\section{Matériel et méthodes}

\section{I. - Animaux}

La population de départ a été décrite précédemment (LECLERCQ et al., I977). Elle est issue de 23 pères et de 93 mères de type Rhode Island appartenant à une population de la Station de Recherches Avicoles de Nouzilly. Les deux lignées sont constituées de façon symétrique en retenant comme coqs des paires de frères ou de demi-frères, l'un étant utilisé pour la lignée grasse, l'autre pour la lignée maigre. On leur associe des poules en opérant de la même façon avec des paires de sœurs; celle qui présente le plus fort gain de poids relatif est choisie pour la lignée "grasse" et celle qui présente le plus faible gain de poids est placée dans la lignée " maigre" ".

Les deux lignées sont donc dès le départ (Fo) très semblables d'un point de vue génétique excepté pour le caractère de l'engraissement. On dispose ainsi de I3 paquets pédigree par lignée réunissant chacun un coq et cinq poules.

Par la suite, chaque génération comporte toujours par lignée I3 parquets de un coq et cinq poules. Les reproducteurs femelles de la génératign Fr sont choisis sur deux critères : le gain de poids relatif au cours des vingt semaines qui suivent le pic de ponte et le nombre d'œufs pondus pendant cette même période.

On choisit les animaux ayant les valeurs les plus intéressantes pour ces deux caractères en fixant un seuil qui est de I/3 d'écart-type au-dessus (lignée grasse) ou au-dessous (lignée maigre) de la moyenne obtenue dans chaque lignée pour le gain de poids. Pour le nombre d'œufs le seuil minimal est fixé à la moyenne. Les différences entre les femelles sélectionnées et l'ensemble des poules de chaque lignée sont présentées dans le tableau I pour les générations $F$ r, F2 et F3. Les coqs sont retenus dans la mesure où ils ont eu au moins quatre sœurs enregistrées et en fonction de la moyenne des performances de ces sœurs pour les deux caractères cités précédemment. La valeur moyenne de ces performances est présentée aussi dans le tableau $I$.

Pour la génération suivante, on procède comme pour la génération précédente si ce n'est que le caractère " nombre d'œufs " est remplacé par le poids d'œufs produit en vingt semaines.

Le tableau I fournit les effectifs d'animaux mis en œuvre à chaque génération.

\section{2. - Conditions d'élevage}

Les poussins sont nourris à volonté jusqu'à l'âge adulte. La durée de la période éclairée est constante de la naissance au pic de ponte : I4 heures pour la première génération, I2 heures pour toutes les suivantes.

Les poulettes sont placées en cages individuelles à 1'âge de $\mathrm{I} 6$ semaines. On leur distribue l'aliment de la période de ponte à partir de l'âge de $\mathrm{I} 8$ semaines. Cet aliment renferme : $62 \mathrm{p}$. Ioo de maïs, ro p. roo de blé, r4 p. roo de tourteau de soja, 3 p. roo de farine de viande, o, I p. roo de méthionine, des minéraux et 


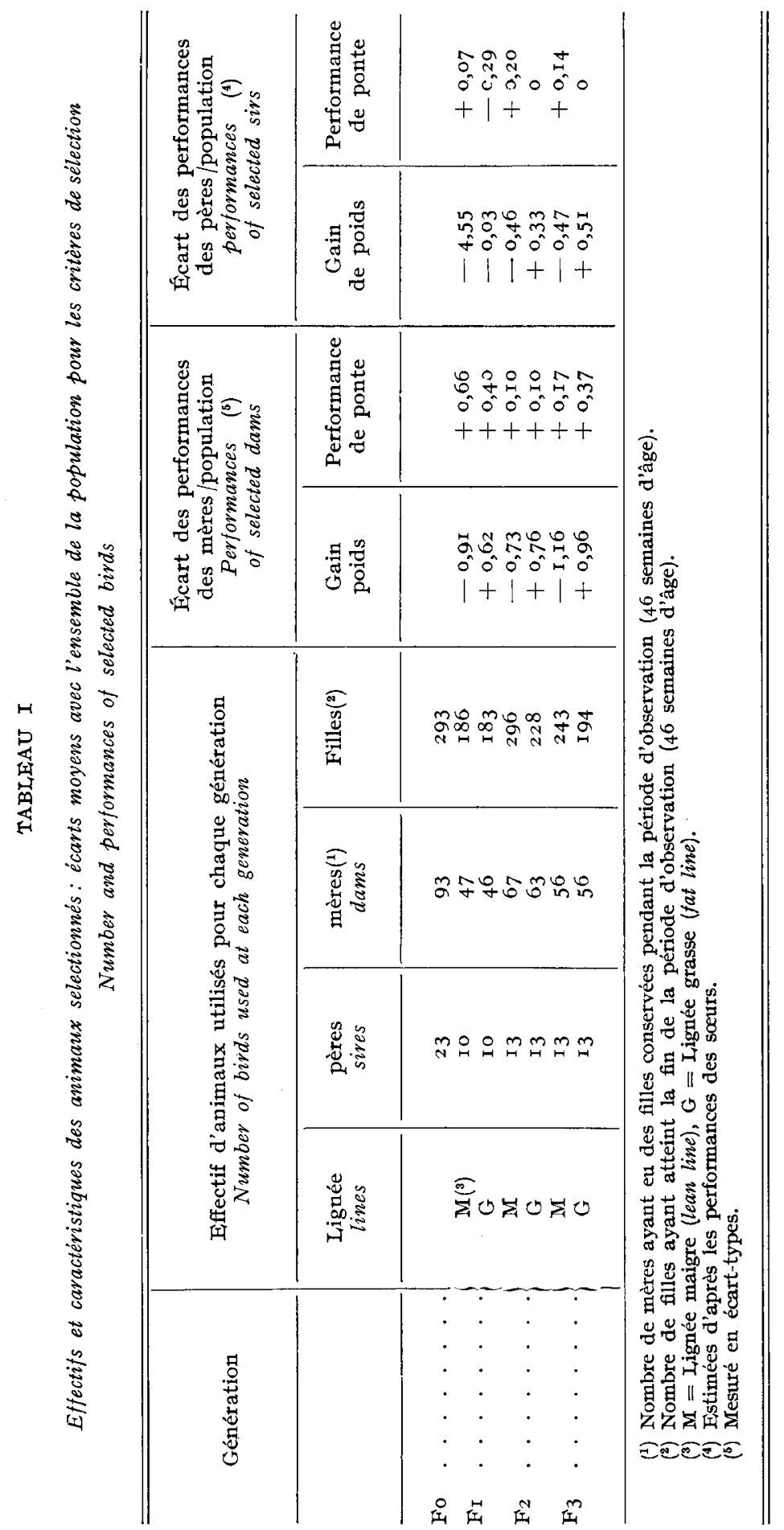


des vitamines. Il est calculé de façon à apporter 2800 Kcal d'énergie métabolisable, I55 $\mathrm{g}$ de protéines, 3,3 $\mathrm{g}$ de méthionine, $7,3 \mathrm{~g}$ de lysine et $30 \mathrm{~g}$ de calcium par $\mathrm{Kg}$.

Après le pic de ponte, la durée de la période journalière d'éclairement est augmentée de 30 minutes par semaine jusqu'à une durée maximum de 16 heures.

\section{3. - Mesures effectuées}

Pour chaque poule, on mesure l'âge lors de la ponte du premier œuf, le nombre et le poids des œufs pondus, ainsi que le poids vif mesuré à différents âges : pic de ponte ( 26 semaines d'âge), 20 semaines plus tard ( 46 semaines d'âge) et en fin de ponte ( 58 semaines d'âge). Le poids des œufs pondus est déterminé périodiquement par pesée de 3 œufs par poule autour des âges de $28,32,36$, 40 et 44 semaines. Les poids vifs sont mesurés en prenant les précautions signalées antérieurement (LECLERCQ et al., I977).

Des animaux de la génération F2 (Io par lignée) sont prélevés à 1'âge de 46 semaines afin de procéder à des mesures de graisse abdominale et de lipides corporels. Ces animaux sont choisis parmi ceux ayant le gain de poids relatif le plus élevé ou le plus faible. Les méthodes de prélèvement et d'analyse ont été décrites par ailleurs (DELPECH et RICARD, I965).

Enfin, des mesures de consommation d'aliment ont été réalisées sur les deux dernières générations. Pour l'avant-dernière, le contrôle a été fait par groupe de 24 poules; pour la dernière génération, on a procédé à un enregistrement individuel sur une partie des animaux : I25 de la lignée grasse et I32 de la lignée maigre. Les contrôles ont eu lieu toutes les quatres semaines entre les âges de 26 à 46 semaines.

\section{4. - Calculs}

L'estimation des héritabilités est basée sur l'analyse hiérarchique de la variance selon la méthode décrite par KING et ANDERSON, (I954). Les écarts-types approchés sont calculés selon celle de BECKER (I964). Un modèle identique est appliqué pour l'analyse de la covariance. Bien que ces analyses aient été réalisés sur tous les caractères, nous ne présentons que les résultats intéressants le plus directement le thème de cette étude.

Pour ce qui concerne les consommations d'aliment, la comparaison compète des deux lignées n'a été possible que pour la dernière génération où les données avaient été recueillies individuellement. Nous avons utilisé la régression multiple reliant la consommation (C) quotidienne d'aliment à la taille métabolique (poids vif élevé à la puissance 0,75 ) et aux poids d'œuf exporté chaque jour (E) :

$$
\mathrm{C}=b_{\mathrm{r}} \mathrm{P}^{0,75}+b_{2} \mathrm{E}+b_{3}
$$

où $b_{1}, b_{2}$ et $b_{3}$ sont des constantes.

Il est ainsi possible de comparer les consommations des deux lignées en tenant compte de leurs différences éventuelles de poids vif et de performances de ponte. Pour le poids vif, nous avons retenu le poids vif au pic qui rend le mieux compte de la masse active (muscle, différents organes). Nous avons aussi utilisé le poids vif moyen (moyenne des poids au pic de ponte et à l'âge de 46 semaines) qui, lui, permet de mieux estimer la surface corporelle. 


\section{Résultats}

Nous avons tenu à démontrer une fois encore que le gain de poids vif pendant la ponte est un bon critère de mesure de l'engraissement des poules pondeuses. Les résultats de l'analyse de la composition corporelle sont présentés dans le tableau 2. Il s'agit non pas d'animaux représentatifs de chaque lignée mais de deux groupes présentant des caractéristiques extrêmes pour le gain de poids relatif. Ces changements de poids sont associés à une évolution parallèle et de grande amplitude de la graisse abdominale et des lipides totaux. Le développement des différents dépôts adipeux (abdomen et carcasse) est étroitement lié. La corrélation entre graisse abdominale et le reste des lipides est élevée $(+0,87)$, surtout pour les animaux à faible gain de poids relatif.

Les performances moyennes enregistrées sur les quatre générations sont rassemblées dans le tableau 3 . Le résultat le plus régulier est l'évolution divergente du gain de poids et du gain de poids relatif sous l'influence de la sélection. D'une génération à 1'autre, la progression est sensible dans chaque lignée. Les résultats obtenus en $\mathrm{F}_{3}$ sont présentés dans la figure $\mathrm{I}$, comme illustration de la divergence réalisée entre lignées. En ce qui concerne cette sélection, on doit cependant remarquer que la population de départ (Fo) a présenté un gain de poids relatif très élevé et jamais retrouvé par la suite; nous aurons l'occasion d'évoquer ce problème au cours de la discussion. Les autres caractères (tabl. 3) sont plus stables. Au fil des générations, le poids vif au pic de ponte varie peu. Il en est de même de la maturité sexuelle (âge moyen au premier œuf) et du poids total d'œufs produit. Pour ce caractère, les différences entre lignées ne sont pas régulières. On note une variation significative pour la maturité sexuelle dans la génération $F_{2}$, qui disparait en $F_{3}$. Les poules de la lignée maigre sont un peu plus légères aux générations $F$ I et $F_{3}$ mais pas en $F_{2}$. Le poids total d'œufs produit n'est pas différent excepté en dernière génération; cette différence est d'ailleurs faible mais significative.

Le tableau 4 fournit les résultats de calcul d'héritabilité du gain de poids relatif au sein des deux lignées et au cours des trois dernières générations; les valeurs observées en génération Fo ont été publiées antérieurement (LEOLERCQ et al., I977). On constate dans les deux lignées une décroissance régulière de l'héritabilité du gain de poids, les valeurs enregistrées en $\mathrm{F}_{3}$ étant particulièrement faibles.

Dans le tableau 5, nous présentons les valeurs des corrélations phénotypiques et génotypiques entre le gain de poids relatif et trois autres caractères : le poids vif au pic de ponte, le poids total d'œufs produit de 26 à 46 semaines d'âge et la maturité sexuelle. Aucune corrélation phénotypique n'atteint une valeur importante; en particulier, la corrélation entre le gain de poids relatif et le poids d'œufs produit est remarquablement proche de 0 . En ce qui concerne les corrélations génotypiques, on note parfois des valeurs élevées pour le poids d'œuf produit mais ce phénomène n'est pas régulier.

Les comparaisons de consommation d'aliment réalisées pour les générations $\mathrm{F}_{2}$ et $\mathrm{F}_{3}$ entre les âges de 26 à 46 semaines font 1 'objet du tableau 6 . Les poules de la lignée maigre consomment toujours moins d'aliment que celles de la lignée grasse et leur efficacité alimentaire est systématiquement meilleure. Cependant pour que la comparaison soit correcte il est indispensable de tenir compte du poids vif des animaux et de l'intensité de leur production d'œufs. Cela n'a pu être obtenu avec la génération $F$, le contrôle de consommation n'ayant pas été fait sur les 
B. LECLERCQ, J. C. BLUM, J. P. BOYER

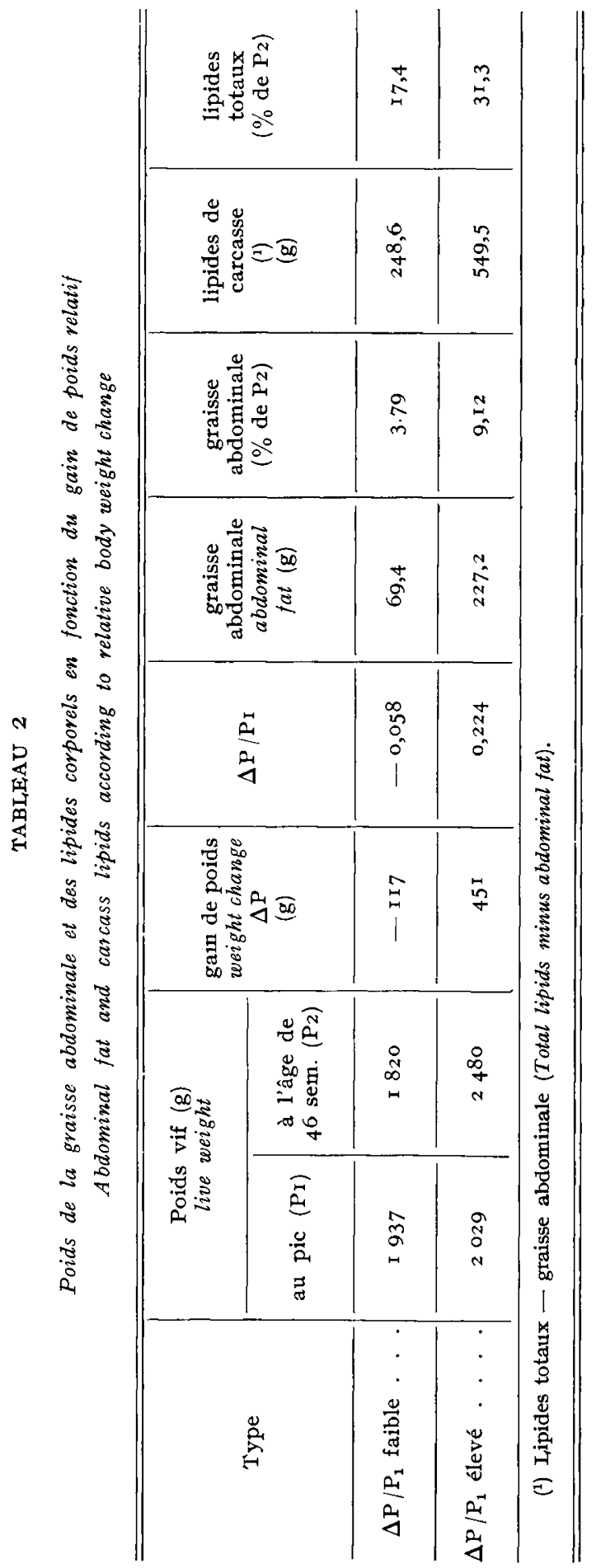




\begin{tabular}{|c|c|c|c|c|c|c|c|c|}
\hline \multirow{4}{*}{$m$} & 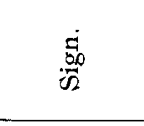 & 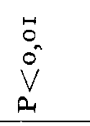 & 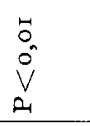 & 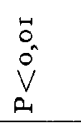 & $\frac{\pi_{2}^{2}}{2}$ & $\begin{array}{l}\text { t. } \\
\text { o. } \\
\text { va }\end{array}$ & & $\begin{array}{l}\vec{b} \\
\dot{0} \\
\dot{v} \\
\Delta\end{array}$ \\
\hline & 密总引总 & 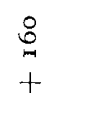 & $\begin{array}{l}\stackrel{\circ}{\circ} \\
+\end{array}$ & 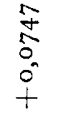 & $\hat{i}$ & 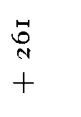 & $\begin{array}{l}3 \\
0^{2} \\
+\end{array}$ & $\begin{array}{l}\text { in } \\
+\end{array}$ \\
\hline & 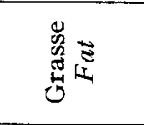 & 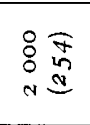 & 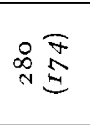 & 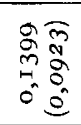 & 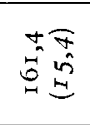 & 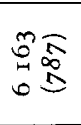 & $\hat{\hat{a}}$ & 胥㺼 \\
\hline & 茄 & 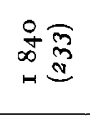 & 於食 & 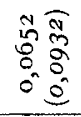 & 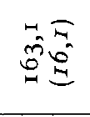 & 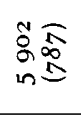 & 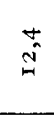 & $\mathbb{J}^{\widehat{S}}$ \\
\hline \multirow{4}{*}{ है } & 离品 & $\frac{\sigma_{2}}{2}$ & 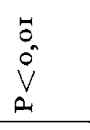 & $\begin{array}{l}b_{0} \\
\hat{V}^{-} \\
\sigma_{1}\end{array}$ & $\begin{array}{l}0 \\
0 \\
0 \\
v \\
\sigma\end{array}$ & 拨 & & \\
\hline & 甾总密密 & I & $\begin{array}{l}8 \\
+ \\
+\end{array}$ & 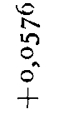 & $\hat{i}$ & $\stackrel{\square}{1}$ & $\begin{array}{l}+ \\
+ \\
+\end{array}$ & \\
\hline & 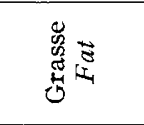 & 容鸽 & 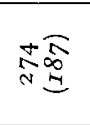 & 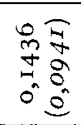 & 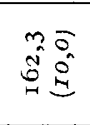 & 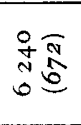 & ज̃ & \\
\hline & 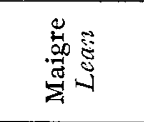 & 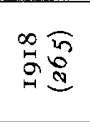 & 㗊蛋 & $\begin{array}{l}: \widehat{y} \\
0.0 \\
000 \\
0.00 \\
0.0\end{array}$ & 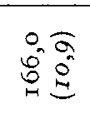 & 品愈 & $\stackrel{\infty}{\circ}$ & \\
\hline \multirow{4}{*}{ 届 } & 咅 & $\begin{array}{l}\vec{o} \\
\text { iv } \\
a \\
\end{array}$ & $\begin{array}{l}\text { : } \\
\text { bे } \\
\text { a }\end{array}$ & 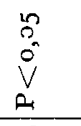 & $\frac{\pi}{\pi}$ & $\tilde{z}_{z}^{2}$ & & \\
\hline & 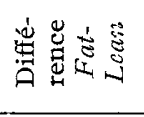 & $\begin{array}{l}\infty \\
\infty \\
+ \\
\end{array}$ & $\stackrel{q}{+}$ & $\begin{array}{l}0 \\
\vdots \\
\vdots \\
\vdots \\
+ \\
+\end{array}$ & $\hat{+}$ & $\stackrel{m}{i}$ & 。 & \\
\hline & 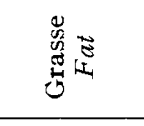 & 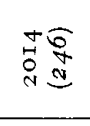 & 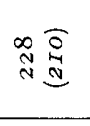 & 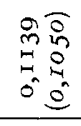 & 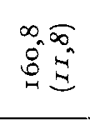 & 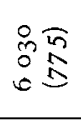 & $\begin{array}{l}\circ \\
\dot{a}\end{array}$ & \\
\hline & 彭 & 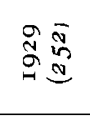 & 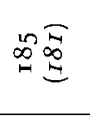 & 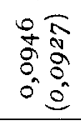 & 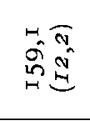 & 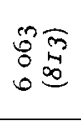 & : & \\
\hline 우 & & \begin{tabular}{l}
$: \bar{z}$ \\
$\vdots$ \\
\hdashline \\
\hdashline
\end{tabular} & 䜣鸽 & 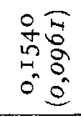 & $\begin{array}{l}\infty \\
\hat{\tilde{r}} \bar{్} \bar{\alpha} \\
\hat{n}\end{array}$ & 융종 & ثे & \\
\hline $\begin{array}{l}\text { 总 } \\
\text { 营 } \\
\text { 总 }\end{array}$ & 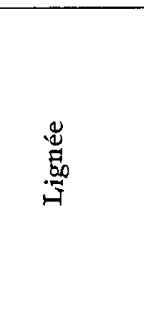 & 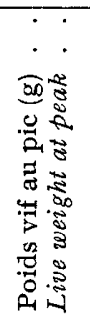 & 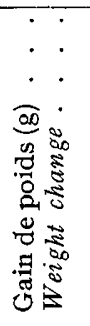 & 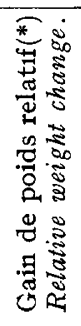 & 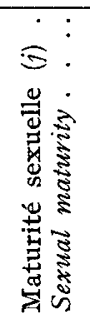 & 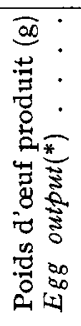 & 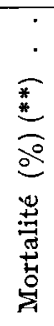 & 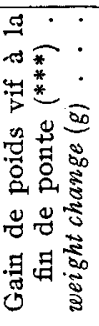 \\
\hline
\end{tabular}


TABLEAU 4

Heritabilité du gain de poids relatif pendant la ponte $(\Delta P / P)$ Heritability of relative body weight change during the laying period

\begin{tabular}{|c|c|c|c|c|}
\hline Génération & $\begin{array}{l}\text { Lignée } \\
\text { Line }\end{array}$ & $\begin{array}{c}h_{p}^{2} \\
\text { sire component }\end{array}$ & $\begin{array}{c}h_{\mathrm{M}}^{2} \\
\text { dam component }\end{array}$ & $h_{p M}^{2}$ \\
\hline FI & $\begin{array}{l}M\left({ }^{1}\right) \\
G\end{array}$ & $\begin{array}{l}0,66(0,4 \mathrm{I})\left({ }^{2}\right) \\
0,55(0,37)\end{array}$ & $\begin{array}{l}\text { o,I } 7(0,26) \\
0,16(0,27)\end{array}$ & $\begin{array}{l}0,4 \mathrm{I}(0,29) \\
0,35(0,26)\end{array}$ \\
\hline $\mathrm{F}_{2}$ & $\begin{array}{l}\mathrm{M} \\
\mathrm{G}\end{array}$ & $\begin{array}{l}0,30(0,20) \\
0,09(0,20)\end{array}$ & $\begin{array}{l}0,09(0,20) \\
0,71(0,75)\end{array}$ & $\begin{array}{l}\mathrm{C}, 22(0,20) \\
0,40(0,22)\end{array}$ \\
\hline $\mathrm{F}_{3}$. & $\begin{array}{l}M \\
G\end{array}$ & $\begin{array}{r}0, \mathrm{I} 4(0, \mathrm{I} 8) \\
-0,03(0, \mathrm{I} 3)\end{array}$ & $\begin{array}{l}0,25(0,26) \\
0,12(0,33)\end{array}$ & $\begin{array}{l}0,20(0,17) \\
0,05(0,08)\end{array}$ \\
\hline
\end{tabular}

(1) $\mathrm{M}=$ Lignée maigre, lean line; $\mathrm{G}=$ Lignée grasse, fat line.

( $\left.{ }^{2}\right)$ I,e nombre entre parenthèses correspond à l'écart-type approché calculé selon la formule de BECKER (1964).

poules prises individuellement. On constate tout de même que pour un poids vif identique, la lignée maigre a mieux pondu en consommant plutôt moins, ce qui conduit à une amélioration de l'indice. Le contrôle individuel mis en place en génération $\mathrm{F}_{3}$ permet en principe une étude plus précise. Nous avons comparé les deux lignées en utilisant l'équation de régression multiple présentée plus haut. Deux hypothèses ont été retenues pour le poids vif : soit le poids au pic qui est un meilleur critère d'estimation de la masse métaboliquement active de l'animal, soit le poids vif moyen qui rend mieux compte de la taille moyenne des animaux et permet une meilleure estimation de leur surface corporelle. Avec la première hypothèse, on aboutit aux deux équations suivantes :

lignée maigre : $\mathrm{C}=0, \mathrm{I} 675 \mathrm{P}^{0,75}+\mathrm{I}, 046 \mathrm{E}+9,6$ avec $\mathrm{R}^{2}=0,60$

lignée grasse : $\mathrm{C}=0,2556 \mathrm{P}^{0,75}+0,7278 \mathrm{E}+\mathrm{I}, \mathrm{I}$ avec $\mathrm{R}^{2}=0,57$

Les cœfficients $0, I 675$ et 0,2556 sont significativement différents $(F=2,26)$; les cœfficients 1,046 et 0,7278 ne le sont pas.

La seconde hypothèse conduit à deux nouvelles équations :

lignée maigre : $\mathrm{C}=0,2185 \mathrm{P}^{0,75}+0,9092 \mathrm{E}+0,4$ avec $\mathrm{R}^{2}=0,72$

lignée grasse : $\mathrm{C}=0,2685 \mathrm{P}^{\mathbf{0 , 7 5}}+0,7048 \mathrm{E}-5,2$ avec $\mathrm{R}^{2}=0,68$

Cette fois, les coefficients de $\mathrm{P}^{0,75}$ et de $\mathrm{E}$ ne sont plus significativement différents. Il en est de même des deux plans de régression multiple illustrant ces deux équations; autrement dit, pendant la génération $F_{3}$, les différences entre les consommations des deux lignées ne sont plus significatives si l'on tient compte des poids vifs et des performances de ponte. 


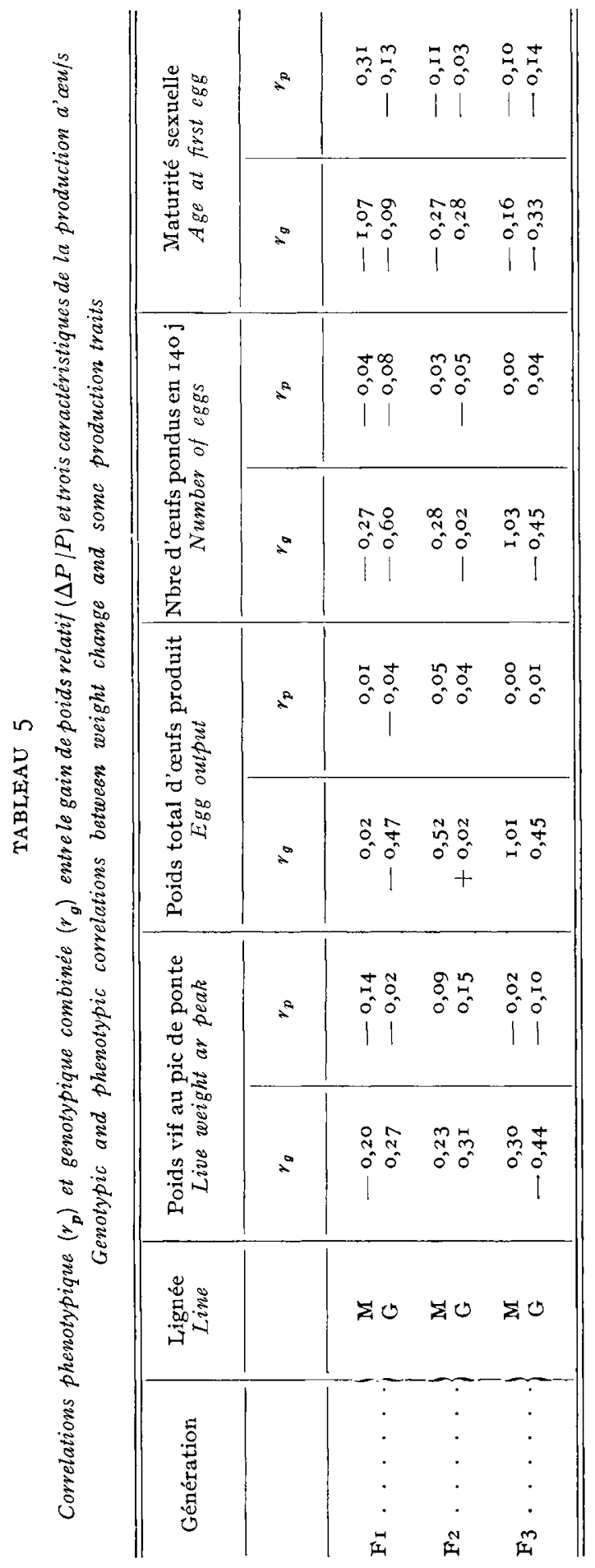




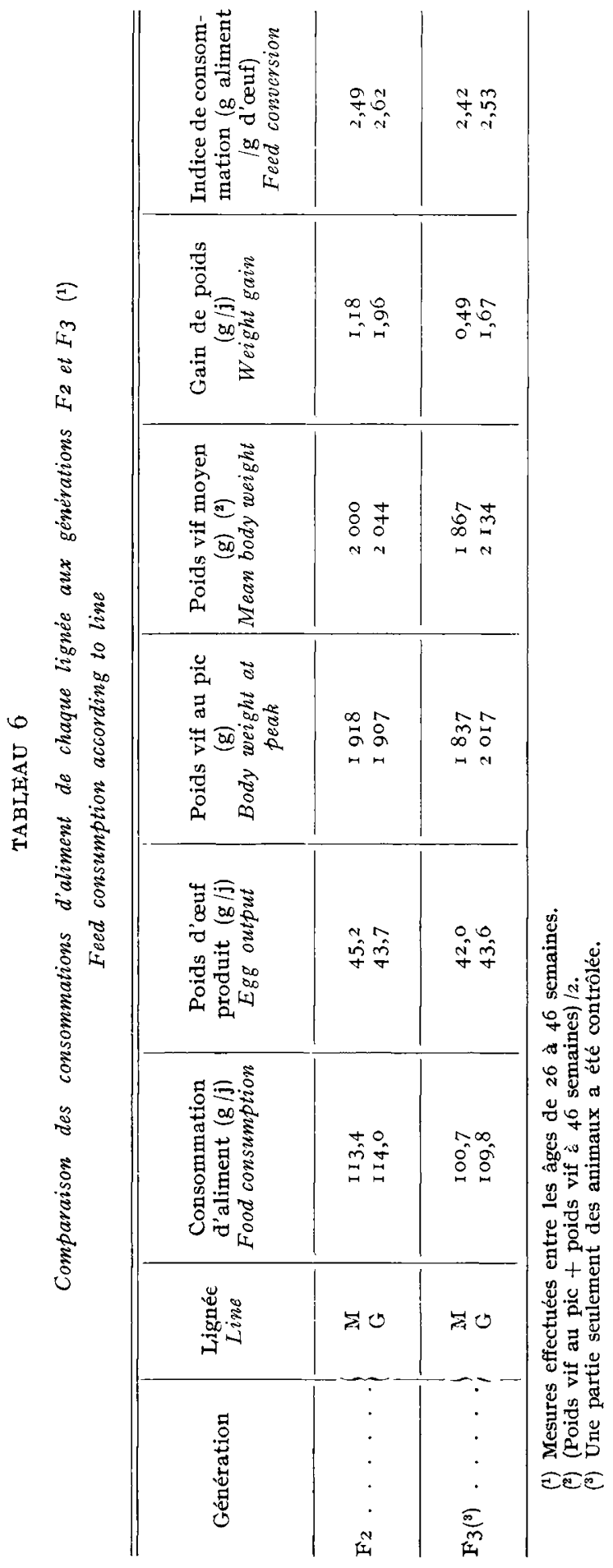




\section{Discussion et conclusion}

Nos résultats démontrent clairement que la sélection des poules adultes pour leur état d'engraissement peut être réalisée par la simple mesure du gain de poids vif. Certes, la valeur moyenne de l'héritabilité de ce caractère est moyenne et a diminué rapidement dans nos conditions. Il n'en demeure pas moins qu'en trois générations nous avons pu obtenir deux lignées significativement différentes. L'engraissement qui se développe au cours de la ponte est donc bien en partie sous la dépendance d'un déterminisme génétique exploitable. Toutefois, comme le montrent les histogrammes de la figure $I$, la variabilité demeure très élevée après trois générations de sélection. Il existe donc des influences importantes du milieu ou des intéractions entre génome et milieu. Ces influences sont encore mal connues. Nous avons eu l'occasion d'étudier quelques unes d'entre elles au
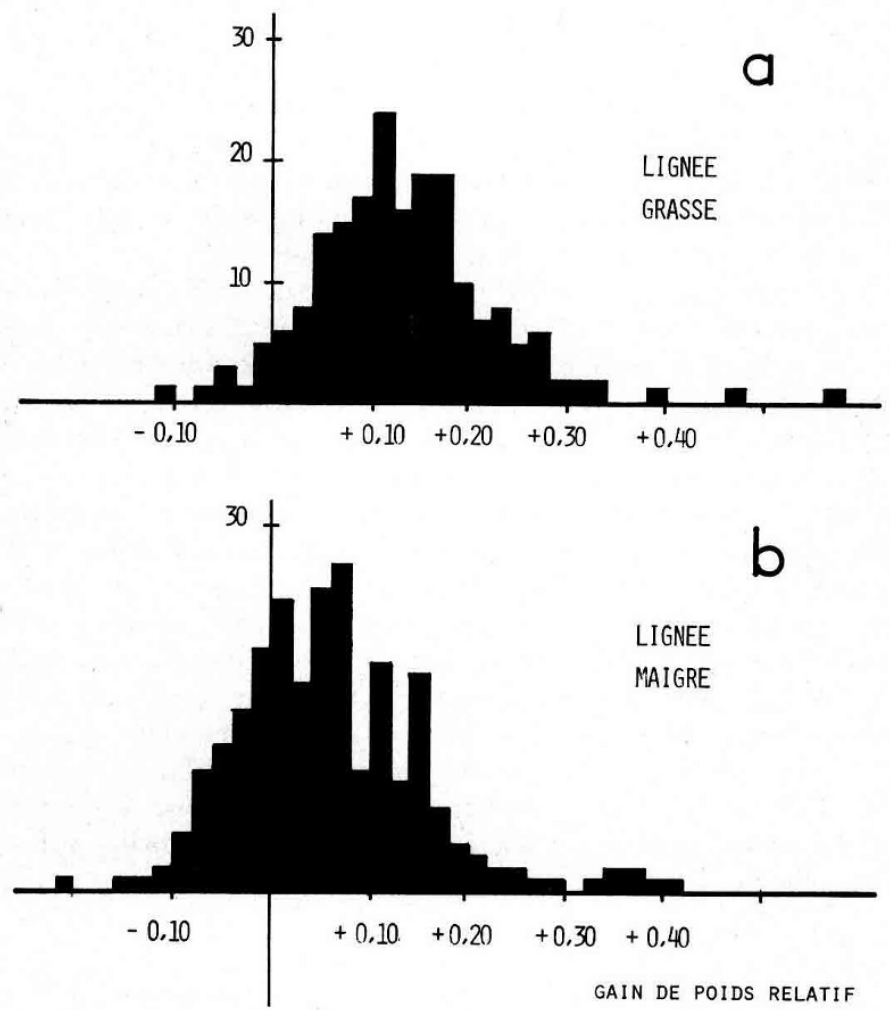

FIG. I. - Répartition des poules en fonction des gains de poids relatifs au cours de la troisième génération dans les deux lignées maigre et grasse

a) lignée grasse.

b) lignée maigre.

Repartition of hens according the relative weight gains during the third generation of selection in two lines: lean and fat.

a) fat line.

b) mean line. 
cours d'une revue bibliographique (LECLERCQ, I977), montrant le rôle de la température ambiante et de l'alimentation. Ces deux facteurs du milieu ont peu de chance de rendre compte des différences observées lors de nos essais puisque les animaux vivaient dans le même local et consommaient un régime pratiquement identique (même formulation). Il faudrait rechercher les autres paramètres du milieu qui influencent l'engraissement. La différence entre le gain de poids de la génération Fo et les suivantes témoigne, elle-aussi d'une intervention notable de facteurs du milieu inconnus pour l'instant.

Il faut en outre remarquer que les différences entre lignées se poursuivent au-delà de 46 semaines d'âge. En effet, la lignée grasse continue d'engraisser plus que la lignée maigre, au cours de la seconde partie de la période de ponte. La propension à déposer des lipides de réserve semble donc un caractère qui se maintient pendant toute la période de reproduction.

Deux conséquences de notre sélection sur l'engraissement doivent être discutées. Il s'agit des relations entre engraissement et performances de ponte d'une part et entre engraissement et efficacité alimentaire d'autre part.

\section{I. - Engraissement et performances de ponte}

Des corrélations trouvées régulièrement nulles entre le gain de poids vif et la ponte, nous pouvons conclure que, chez la pondeuse de format léger ou moyen, il n'y a pas de relation entre ces deux caractères.

Une seule observation contredit cette conclusion. Il s'agit de la différence de poids d'œuf produit entre les deux lignées à la génération $\mathrm{F}_{3}$ en défaveur des poules maigres. Ce résultat peut provenir d'un effet d'échantillonnage dû au faible nombre de parquets pédigree mis en place. De toute façon, cotte différence est faible et ne correspond pas à l'accentuation d'une tendance qui auraiť été amorcée lors des générations précédentes.

Donc, dans les conditions d'environnement (ambiance climatisée, nycthémères de durée constante) et d'alimentation (apports suffisants ou phéthoriques de nutriments) de l'élevage moderne, les réserves grasses ne semblent jouer aucun rôle. Elles ne représentent ni un avantage, ni une gêne pour la pondeuse, tout au moins chez celles qui appartiennent aux souches légères ou de taille moyenne. Nous confirmons nos premières observations (LECLERCQ et al., I977) ainsi que celles d'autres auteurs (Bordas et MERAT, I976; WATANABE et al., I975; NEIL, et al., I977). I1 conviendrait d'effectuer une étude semblable chez les reproductrices lourdes, sachant que leur engraissement excessif est jugé responsable de performances de ponte médiocres du fait surtout d'ovulations intrapéritonéales (JAAP et MUIR, I968).

\section{2. - Engraissement et efficacité alimentaire}

La réduction des dépôts lipidiques de réserves pendant la ponte doit entrainer théoriquement une économie d'aliment. Compte-tenu du rendement des synthèses lipidiques (GRIMBERGEN, I974), on peut estimer qu'il faut I,25 Kcalorie d'énergie métabolisable par Kcalorie mise en réserve. Lorsque la valeur énergétique est de $2800 \mathrm{Kcal} / \mathrm{kg}$ comme dans le présent essai, l'économie théorique d'aliment devrait être de 4,2 $\mathrm{g}$ par $\mathrm{g}$ de gain de poids, en supposant que tout le gain de poids est constitué de lipides. 
Au cours de nos essais, une telle économie n'a pu être mesurée avec précision. En effet, si en génération F2 les poules maigres paraissent bien plus efficaces que les poules grasses, la comparaison n'est pas très facile en $F_{3}$. L'un des problèmes rencontrés concerne le mode d'expression du poids vif. Ce caractère influence notablement la consommation et l'efficacité alimentaire, à tel point que plusieurs expériences de sélection sur la seule efficacité alimentaire ont entrainé avec 1'amélioration de ce caractère, une réduction notable du poids vif (Usur et al., I979; Harris, I 969). Dans notre essai, si l'on utilise pour les calculs le poids vif au pic de ponte, la souche maigre se distingue de la souche grasse par un besoin spécifique d'entretien significativement plus faible. La comparaison des deux lignées ne peut guère être poursuivie, les deux plans de régression n'étant pas parallèles. Si l'on utilise le poids vif moyen, il n'existe plus de différence significative entre lignées, bien qu'une tenđance subsiste en faveur des maigres. L'économie réalisée par ces dernières est en fait trop faible par rapport aux erreurs expérimentales inéluctables. La valeur plus élevée des coefficients de régression multiple lorsque l'on fait appel au poids vif moyen conduit à penser que cette mesure rend en définitive mieux compte des dépenses d'entretien. La corrélation entre engraissement et efficacité alimentaire semble donc bien exister chez la poule en ponte, comme l'ont par ailleurs observé d'autres auteurs (HAGGER, I978; NEIL et al., I978; MORISSON et LEESON, I978). Toutefois, cette corrélation n'explique qu'une proportion modeste des différences d'efficacité alimentaire; une part plus importante de la variance revient très probablement aux dépenses spécifiques d'entretien comme le suggèrent MORRISON et LEESON (I978).

En conclusion, la sélection contre l'engraissement est possible et efficace. Cependant, 1'héritabilité de ce caractère semble diminuer rapidement. La découverte de nouveaux facteurs du milieu exerçant une influence notable sur le gain de poids, devrait permettre d'imaginer des conditions d'élevage plus précises permettant de mieux mettre en évidence les différences d'ordre génétique. De toute façon, la prise en compte de ce seul critère ne garantit pas d'obtenir les meilleurs résultats d'efficacité alimentaire. Il faut lui associer une sélection sur ce dernier caractère, qui ,à elle seule, n'est pas non plus totalement efficace pour obtenir des animaux maigres.

Reçu pour publication en janvier $198 \mathrm{I}$.

\title{
Summary
}

\author{
Influence of a modification in increasing of fat deposit \\ in the laying Hen: resultats of a divergent selection on 3 generations
}

Two lines of medium size laying hens were selected over three generations for either a low or a high relative body weight gain during the laying period ( 26 to 46 weeks of age). In both lines this character was susceptible to the effects of selection but the heritabilities diminished sharply. No significant correlation was observed between the relative body weight change (as an index of fattening) and the laying performances. In the lean line (low body weight change), feed efficiency was superior to that of the fatty line; however, the differences were not very pronounced. It seems that the best way of improving food efficiency in laying hens would be to select for body weight change and food conversion in parallel. 


\section{Références bibliographiques}

BECKER W. A., I964. Manual of procedures in quantitative genetics. 70 p. Washington state University, Pullman.

Bordas A., MERAT P., I976. Gain de poids vif pendant la ponte et sélection sur l'efficacité alimentaire chez la poule. Ann. Génét. Sél. anim. 8, 527-536.

DELPECH P., RICARD H. F., I965. Relation entre les dépôts adipeux viscéraux et les lipides corporels chez le poulet. Ann. Zootech., 14, I8I-I89.

GRIMBERGEN A. H. M., I974. Energy expenditure under productive conditions. In Energy requirement of poultry. British Poultry Science Ltd Edit. Edinburgh.

HAGGER C., I978. Untersuchungen zur Futterverwertung von Leghenen. 2. Teil : Genetische Parameter. Arch. Geflügelk., 42, ro-I5.

HARRIS P. C., I969. Effectiveness of the egg mass/feed consumption ratio in improving genetic productive efficiency of laying hens. Poult. Sci., 48, I8 I 7 .

KING S. C., HENDERSON C. R., I954. Variance components analysis in héritability studies. Poult. Sci., 33, 147-154.

JAAP R. G., MUIR F. V., 1968. Erratic ovipositions and egg defects in broiler type pullets. Poult. Sci., 47, 417-423.

LECLERCQ B., 1975. Determination simultanée et in vivo des intensités de dépôts et de mobilisation des acides gras du tissu adipeux de la poule pondeuse. Influence du rationnement. Ann. Biol. anim. Bioch. Biophys., 15, 63-71.

LECLERCQ B., I977. La composition corporelle de la poule et ses facteurs de variation. In La composition corporelle des volailles. I.N.R.A. Edit.

LECLERCQ B., BLUM J. C., BOYER J. P., 1977. Signification and genetic control of body weight change in the hen during the laying period. Arch. Geflügelk, 41, I21-124.

MORRISON W. D., LEESON S., I978. - Relationship of feed efficiency to carcass composition and metabolic rate in laying birds. Poult. Sci., 57, 735-939.

NeIL, A. R., ReIchmann K. G., KonNor J. K., I977. Biochemical, physiological and production indices related to fat metabolism in the laying fowl at various stages of physiological development. Brit. Poult. Sci., 18, 3I5-324.

Usui K., Sakamoto M., IIsuka Y., Muto A., Yamada Y., 1979. Index selection for improving feed efficiency. Jap. Poult. Sci., 16, 26-34.

WATANABE K., YAMADA Y., EBISAWA S., YAMAdA Y., I975. Breeding for improvement of feed efficiency in egg layers. I. Estimation of genetic parameters. Jap. Poult. Sci., 12, 21 9-229 